\title{
The Journal of the Brazilian College of Surgeons
}

\section{A Revista do Colégio Brasileiro de Cirurgiōes}

\author{
Guilherme Pinto Bravo Neto, TCBC-RJ'
}

$T^{\text {Thersen }}$ he Journal of The Brazilian College of Surgeons (CBC), published uninterruptedly since 1974 , has been gaining greater visibility in recent years, since its indexing in the electronic library SciELO (Scientific Electronic Library Online) in 2005, and later in Medline/PubMed and other databases. Our articles have been read worldwide and quoted in several international publications of greatest impact, and our authors, contacted by editors of international journals to serve as ad hoc reviewers in several works, based on the expertise inferred through their publications on our journal. Our impact factor measured by the JCR (Journal Citation Reports) has been steadily increasing, and nationally, we reached the Qualis B1 level at CAPES (Higher Education Personnel Improvement Coordination), a degree now stamped on the cover of our journal. We are moving forward, but we still have much work ahead. More pressingly, we are striving to achieve more rapid assessments of the works sent and trying to substantially reduce the time for definition of approval, rejection or request of modifications suggested by our reviewers. For this, we rely on the promptness and seriousness of our editorial board, which will gain new signings in the coming months. Soon works submission will be done exclusively through our submission online platform, already in place and that can be accessed through the $C B C$ website, which should further streamline the evaluation and review process and give greater transparency to the authors to monitor the progress of their work. We hope that in the first half of this year we managed to catch up on our publications and the journal can be accessed in full on the SciELO database and CBC site even during the last month of the bimonthly journal period. And that the printed journal reaches subscribers and $C B C$ members already in the first days following the last month's cover. These perspectives are due to not only our respect and consideration to our authors and readers, but also to the guidelines set for maintenance of indexations already achieved and for approval in other search electronic platforms, such as PubMed Central, which has been in negotiation for a few months now. On the other hand, greater visibility and impact also require greater scientific quality of articles published and therefore higher rigor in assessments by the editorial board. We know of the difficulties in conducting scientific work in our environment, particularly clinical trials. Inefficient patients referencing policy dilute the experience with certain diseases and reduce treatment effectiveness and degraded hospitals scrapped, especially university ones, are some of the many obstacles to be faced by Brazilian researchers, particularly by the surgeon ones. And once overcome the barriers to achieving the original work, one still need to deal with the difficulties in its publication, especially when opting for high-impact journals. Besides the indisputable quality of work, one needs an adequate translation into English, preferably by professionals experienced with the scientific language, and a renowned origin of institutions and authors. All these minutiae greatly hamper the publication possibilities for the most active surgeons, with all their professional activities, which often interfere even in their own family gatherings. On the other hand, in the academic world, the need to publish papers is increasing, which has generated serious distortions between professionals of the same university as it relates to clinical practice and scientific production, a ratio that is becoming contradictorily antagonistic. Unfortunately, we cannot change this reality, but we encourage our members to invest in quality clinical research, able to promote and extol their primary surgical activity. These difficulties have been chronically reflected in the types of articles we receive: largely case reports and review articles, to the detriment of quality original articles, the latter composed mostly by experimental work and master's and doctorate degree theses. For publication of these numerous Case Reports, the CBC has an online journal dedicated exclusively to these articles and videos, the Journal of Surgical Case Reports, which can be accessed through the CBC website and soon will also feature its own ISSN (International Standard Serial Number). We also have specialized translators who translate the work into English, as yet with no cost to the authors. We therefore believe that the CBC journal can not only further enhance and contribute to the dissemination of high quality scientific articles, but also stimulate our surgeons to develop their scientific and investigative potential, with the certainty of a good welcome in their home, the Brazilian College of Surgeons.

1. Departamento de Cirurgia Universidade Federal do Rio de Janeiro (UFRJ), Rio de Janeiro, RJ, Brasil. 